\title{
SIMILARITY OF MATRICES OVER FINITE RINGS
}

\author{
J. POMFRET
}

ABstract. It is shown that questions of similarity of certain invertible matrices over a finite ring can be reduced to questions of similarity over finite fields through the application of canonical epimorphisms.

Suprunenko has shown in [3] that two invertible matrices over $Z / Z_{m}$ whose orders are relatively prime to $m$ are similar if and only if their canonical images are similar over the fields $Z \mid Z p$ for each prime divisor $p$ of $m$. An analogous result holds for invertible matrices over any finite commutative ring with identity.

Preliminaries. If $R$ is a finite commutative ring with identity, then $R$ is uniquely a ring direct product of finite local rings [1, Theorem 8.7, p. 90]. Suppose that $R=\prod_{i=1}^{t} R_{i}$, where $R_{i}$ is a finite local ring with maximal ideal $M_{i}$. Each $R_{i}$ has cardinality $p_{i}^{e_{i}}$ for some prime $p$ and has associated with it a canonical projection,

$$
h_{i}: R_{i} \rightarrow R_{i} / M_{i}=\mathrm{GF}\left(p_{i}^{f_{i}}\right) .
$$

Setting $k_{i}=\mathrm{GF}\left(p_{i}^{f_{i}}\right)$ we will say that the finite fields $\left\{k_{i}: i=1,2, \cdots, t\right\}$ are the fields associated with $R$.

Observe that the decomposition of $R$ carries over to the general linear group of degree $n$ over $R$ yielding $\mathrm{GL}_{n}(R) \cong \prod_{i=1}^{t} \mathrm{GL}_{n}\left(R_{i}\right)$. Furthermore, for each $i$, the projection $h_{i}$ induces an epimorphism,

$$
h_{i}: \mathrm{GL}_{n}\left(R_{i}\right) \rightarrow \mathrm{GL}_{n}\left(k_{i}\right) \text {. }
$$

If $\mathrm{GL}_{n}\left(R_{i}\right)$ is taken as the group of $n$ by $n$ invertible matrices over $R_{i}$, then $h_{i}$ is simply reduction modulo $M_{i}$. Note that the kernel of $h_{i}, K_{i}$, has cardinality a power of $p_{i}$ and thus is a solvable group.

The following corollary to P. Hall's extension of the Sylow theorems [2, Theorem 9.3.1, p. 141] is the key result needed for Theorems 1 and 2.

Observation. Let $G$ be a finite group with solvable normal subgroup $K$ and let $\bar{G}=G \mid K=\{\bar{g} \mid g \in G\}$. Let $\alpha$ and $\beta$ be elements of $G$ with $(|\alpha|,|k|)=$ $1=(|\beta|,|K|)$. Then $\bar{\alpha} \sim \bar{\beta}$ implies $\alpha \sim \beta$.

Received by the editors April 12, 1972.

AMS (MOS) subject classifications (1970). Primary 13H99, 15A33, 15A21, 20D20, $20 \mathrm{H} 25$.

Key words and phrases. Similarity, finite local ring, finite solvable group.

(C) American Mathematical Society 1973 
Proof. Since $\bar{\alpha}=\bar{\gamma}^{-1} \bar{\beta} \bar{\gamma}$ for some $\gamma$ it follows that $\langle\alpha\rangle K=\left\langle\gamma^{-1} \beta \gamma\right\rangle K$. By P. Hall's theorem it follows that $\langle\alpha\rangle$ and $\left\langle\gamma^{-1} \beta \gamma\right\rangle$ are conjugate in $\langle\alpha\rangle K$. Thus there is a $\mu$ in $K$ and $r>0$ such that $\mu^{-1} \gamma^{-1} \beta \gamma \mu=\alpha^{r}$. Hence $\bar{\alpha}^{r}=\bar{\gamma}^{-1} \bar{\beta} \bar{\gamma}=\bar{\alpha}$ and, since $\alpha$ and $\bar{\alpha}$ have the same order, $\alpha=\alpha^{r}$. Therefore $\alpha=(\gamma \mu)^{-1} \beta(\gamma \mu)$ and $\alpha \sim \beta$.

The theorems.

THEOREM 1. Let $R$ be a finite local ring with maximal ideal $M$ and $R / M=\mathrm{GF}\left(p^{f}\right)=k$. Let $\alpha, \beta$ be elements of $\mathrm{GL}_{n}(R)$ with $(|(\alpha)|, p)=1$ and $(|(\beta)|, p)=1$. Then $\alpha$ is similar to $\beta$ if and only if $\alpha$ is similar to $\beta$ modulo $M$.

Proof. This follows from the Observation by noting that the kernel, $K$, of $h: \mathrm{GL}_{n}(R) \rightarrow \mathrm{GL}_{n}(R / M)$ is solvable with cardinality a power of $p$.

THEOREM 2. Let $R$ be a finite commutative ring with identity and let the cardinality of $R$ be $m$. Two elements $\alpha$ and $\beta$ of $\mathrm{GL}_{n}(R)$ satisfying $(|(\alpha)|, m)=(|(\beta)|, m)=1$ are similar if and only if their canonical images over the Galois fields associated with $R$ are similar.

Proof. This follows from Theorem 1 directly by means of the sequence of epimorphisms

$$
\mathrm{GL}_{n}(R)=\prod_{i=1}^{t} \mathrm{GL}_{n}\left(R_{i}\right) \stackrel{\pi_{i}}{\longrightarrow} \mathrm{GL}_{n}\left(R_{i}\right) \stackrel{h_{i}}{\longrightarrow} \mathrm{GL}_{n}\left(k_{i}\right) \text {. }
$$

\section{BIBLIOGRAPHY}

1. Michael F. Atiyah and I. G. MacDonald, Introduction to commutative algebra, Addison-Wesley, Reading, Mass., 1969, p. 90 . MR 39 \#4129.

2. Marshall Hall, Jr., The theory of groups, Macmillan, New York, 1959. MR 21 \#1996.

3. D. A. Suprunenko, On the conjugacy of matrices over a ring of residues, Dokl. Akad. Nauk USSR 8 (1964), 693-695. (Russian) MR 30 \#3102.

Department of Mathematics, Clemson University, Clemson, South Carolina 29631

Current address: Department of Mathematics, Bloomsburg State College, Bloomsburg, Pennsylvania 17815 